\title{
On weakly $S$-prime ideals of commutative rings
}

\author{
Fuad Ali Ahmed Almahdi, El Mehdi Bouba and Mohammed \\ Tamekkante
}

\begin{abstract}
Let $R$ be a commutative ring with identity and $S$ be a multiplicative subset of $R$. In this paper, we introduce the concept of weakly $S$-prime ideals which is a generalization of weakly prime ideals. Let $P$ be an ideal of $R$ disjoint with $S$. We say that $P$ is a weakly $S$-prime ideal of $R$ if there exists an $s \in S$ such that, for all $a, b \in R$, if $0 \neq a b \in P$, then $s a \in P$ or $s b \in P$. We show that weakly $S$-prime ideals have many analog properties to these of weakly prime ideals. We also use this new class of ideals to characterize $S$-Noetherian rings and $S$-principal ideal rings.
\end{abstract}

\section{Introduction}

Throughout this paper all rings are commutative with $1 \neq 0$. An ideal $I$ of a ring $R$ is said to be proper if $I \neq R$. Let $R$ be a ring and $I$ be an ideal of $R$. Then, $\sqrt{0}$ denotes the nilradical of $R$ and $\sqrt{I}$ denotes the radical of $I$.

Recall that a proper ideal $\mathfrak{q}$ of a ring $R$ is said to be prime if, whenever $a, b \in R$ with $a b \in \mathfrak{q}$, then $a \in \mathfrak{q}$ or $b \in \mathfrak{q}$. The prime ideals play a very important role in the commutative rings theory so this notion has been generalized and studied in several directions. The importance of some of these generalizations is same as the prime ideals. For instance, in 2003, D.D. Anderson and E. Smith [1] defined the weakly prime, that is a proper ideal $P$ of $R$ such that for all $a, b \in R$ with $0 \neq a b \in P, a \in P$ or $b \in P$. Every proper prime ideal of $R$

Key Words: $S$-prime ideals, Weakly $S$-prime ideals, $S$-Noetherian rings, Nagata idealization. 2010 Mathematics Subject Classification: Primary 13A15; Secondary 13B02, $13 E 99$.

Received: 11.12 .2020

Accepted: 19.01.2021 
is weakly prime. However, the converse is not true. For instance, (0) is always weakly prime of $R$, and it is prime if and only if $R$ is an integral domain. In a very recent paper[8], the authors introduced the notion of $S$-prime ideal which is also a generalization of prime ideals. As in [8], let $S \subseteq R$ a multiplicative subset of $R$ and $P$ an ideal of $R$ disjoint with $S$, then $P$ is said to be $S$-prime $P$ if there exists an $s \in S$ such that for all $a, b \in R$ with $0 \neq a b \in P, s a \in P$ or $s b \in P$. Note that every (weakly) prime ideal of $R$ which is disjoint with $S$ is an $S$-prime ideal. However, the converse is not true in general [8, Example 1].

The main purpose of this paper is to introduce and to investigate the notion of $S$-prime ideals which is also a generalization of (weakly) prime ideals. Let $R$ be a commutative ring and $S \subseteq R$ be a multiplicative set of $R$. An ideal $P$ of $R$ satisfying $P \cap S=\emptyset$ is said to be weakly $S$-prime if there exists an element $s \in S$ such that, whenever $a, b \in R, 0 \neq a b \in P$ implies $s a \in P$ or $s b \in P$. In this case, we say that $P$ is associated to $s$. Among many results in this paper, it is shown that if $P$ is a weakly $S$-prime ideal of $R$ which is not $S$-prime, then

$P^{2}=0$. Theorem 7 proves that an ideal $P$ is weakly $S$-prime of $R$ (associated to $s \in S)$ if and only if for each $x \notin(P: s)$ we have either $(P: x) \subseteq(P: s)$ or $(P: x)=(0: x)$. In the case when $S$ is consisting only of regular element, it is proved that $P$ is a weakly $S$-prime ideal of $R$ (associated to $s \in S$ ) if and only if $(P: s)$ is a weakly $S$-prime ideal of $R$ (associated to $s \in S$ ) if and only if $S^{-1} P$ is a weakly prime ideal of $S^{-1} R$ and $S^{-1} P \cap R=(P: s)$.

Let $R$ be a commutative ring and $S$ a multiplicative subset of $R$. Recall from[1] that an ideal $I$ of $R$ is called $S$-finite (resp., $S$-principal) if $s I \subseteq J \subseteq I$ for some finitely generated (resp., principal) ideal $J$ of $R$ and some $s \in S$. Also, $R$ is called $S$-Noetherian (resp., $S$-PIR) if each ideal of $R$ is $S$-finite (resp., $S$-principal). Theorem 18 shows that $R$ is $S$-Noetherian (resp., $S$ - $P I R$ ) if and only if every weakly $S$-prime ideal of $R$ is $S$-finite (resp., $S$-principal). Let $R:=R_{1} \times R_{2}, S=S_{1} \times S_{2}$, where $S_{i}$ is a multiplicative subset of $R_{i}$ and $P_{1}$ and $P_{2}$ be an nonzero ideals of $R_{1}$ and $R_{2}$, respectively. Theorem 24 proves that weakly $S$-prime of $R$ coincide with $S$-prime of $R$, and then characterizes them. Hence, we deduce that if, over a ring $R=R_{1} \times R_{2}$, every proper ideal is weakly $S$-prime then $R_{1}$ and $R_{2}$ are fields. We turn off this paper, with a study of some kind of weakly $S$-prime ideals of the idealization of Nagata and the duplication of a ring along an ideal.

\section{Weakly $S$-prime ideals of commutative rings}

Definition 1. Let $R$ be a commutative ring and $S \subseteq R$ be a multiplicative set of $R$. An ideal $P$ of $R$ satisfying $P \cap S=\emptyset$ is said to be weakly $S$-prime if there exists an element $s \in S$ such that, whenever $a, b \in R, 0 \neq a b \in P$ 
implies $s a \in P$ or $s b \in P$. In this case, we say that $P$ is associated to $s$.

Obviously, an $S$-prime ideal of $R$ is weakly $S$-prime. However, the next example shows that the converse implication is not true in general.

Example 2. Set $R:=\mathbb{Z} / 12 \mathbb{Z}$ and $S=\{\overline{3}, \overline{9}\}$. The ideal $(\overline{0})$ is weakly $S$ prime. Now, $\overline{2} \cdot \overline{6}=\overline{0}$. However, $\overline{2} \cdot \overline{3}=\overline{2} \cdot \overline{9}=\overline{6} \cdot \overline{3}=\overline{6} \cdot \overline{9}=\overline{6} \neq \overline{0}$. Hence, ( $\overline{0})$ is not $S$-prime.

A weakly-prime ideal $P$ of $R$ is weakly $S$-prime for each multiplicative set $S$ of $R$ such that $P \cap S=\emptyset$. Conversely, if $S \subseteq U(R)$ is a multiplicative set of $R$ then weakly $S$-prime ideals are just the weakly-prime ideals of $R$. In particular, if $(R, \mathfrak{m})$ is a local ring and $P$ is a proper ideal of $R$, then $P$ is a weakly prime ideal of $R$ if and only if $P$ is a weakly $(R \backslash \mathfrak{m})$-prime ideal of $R$. However, in general, weakly prime ideals and weakly $S$-prime ideals are different concepts as shown by the following example.

Example 3. Set $R:=\mathbb{Z}[X]$ and $S=\left\{2^{n} \mid n \in \mathbb{N}\right\}$. By [8, Example 1], $P=4 X \mathbb{Z}[X]$ is an $S$-prime ideal of $R$, and so weakly $S$-prime. Moreover, $0 \neq 4 X \in P$ but neither $4 \in P$ nor $X \in P$. Thus, $P$ is not weakly prime.

Proposition 4. Let $R$ be a ring, $S$ be a multiplicative subset of $R$, and $P$ be a weakly $S$-prime ideal of $R$. If $P$ is not $S$-prime, then $P^{2}=0$.

Proof. There exists $s \in S$ such that, whenever $a, b \in R, 0 \neq a b \in P$ implies $s a \in P$ or $s b \in P$. Suppose that $P^{2} \neq 0$. We claim that $P$ is $S$-prime.

Let $a, b \in R$ such that $a b \in P$. If $0 \neq a b \in P$, then $s a \in P$ or $s b \in P$. Now, suppose that $a b=0$. If $a P \neq 0$, there is $p \in P$ such that $0 \neq a p$, and so $0 \neq a p=a(p+b) \in P$. Hence, $s a \in P$ or $s(p+b) \in P$. Hence, $s a \in P$ or $s b \in P$. Similarly, if $b P \neq 0$, we obtain the same result. Finally, assume that $a P=0$ and $b P=0$. Since, $P^{2} \neq 0$, there exists $p, q \in P$ such that $p q \neq 0$. Thus, $0 \neq p q=(a+p)(b+q) \in P$. Then, $s(a+p) \in P$ or $s(b+q) \in P$. Therefore, $s a \in P$ or $s b \in P$. Consequently, we conclude that $P$ is an $S$-prime ideal of $R$, as desired.

Corollary 5 ([1], Theorem 1$)$. Let $P$ be a weakly prime ideal of ring $R$. If $P$ is not prime, then $P^{2}=0$.

Proof. It suffices to take $S=\{1\}$ in Proposition4.

Seeing [8, Proposition 2(4)], it is clear that if $P$ is an $S$-prime ideal of $R$ for some multiplicative subset $S$ of $R$, then there exists an $s \in S$ such that $s \sqrt{0_{R}} \subseteq P$. Hence, using Proposition 4, we can deduce easily the following corollary. 
Corollary 6. Let $R$ be a ring, $S$ be a multiplicative subset of $R$, and $P$ be a weakly $S$-prime ideal of $R$. Then, $P \subseteq \sqrt{0_{R}}$ or $s \sqrt{0_{R}} \subseteq P$ for some $s \in S$. In particular, if $R$ is reduced then $P=(0)$ or $P$ is $S$-prime.

Theorem 7. Let $R$ be a ring, $S \subseteq R$ be a multiplicative subset and $P$ an ideal of $R$ disjoint with $S$. Then the following assertions are equivalent:

1. $P$ is weakly $S$-prime of $R$.

2. There exists $s \in S$ such that for each $x \notin(P: s)$ we have either ( $P$ : $x) \subseteq(P: s)$ or $(P: x)=(0: x)$.

3. There is an $s \in S$, such that for all $I$ and $J$ two ideals of $R$, if $0 \neq I J \subseteq$ $P$, then $s I \subseteq P$ or $s J \subseteq P$.

Proof. (1) $\Rightarrow(2)$ There exists $s \in P$ such that, whenever $x, y \in R, x y \in P$ implies $s x \in P$ or $s y \in P$.

Let $x \in R \backslash(P: s)$ (that is $s x \notin P)$ and suppose that $(P: x) \neq(0: x)$. Since $(0: x) \subseteq(P: x)$, there exists $a \in(P: x)$ with $a x \neq 0$. Thus, $0 \neq a x \in P$. Hence, since $s x \notin P$, we have as $\in P$. Now, let $y \in(P: x)$. We have $x y \in P$. If $x y \neq 0$ then $s y \in P$, and so $y \in(P: s)$. Now, suppose that $x y=0$. We have $0 \neq x a=x(a+y) \in P$. Then, $s(a+y) \in P$. Therefore, $s y \in P$. So, $y \in(P: s)$. Consequently, $(P: x) \subseteq(P: s)$.

$(2) \Rightarrow(1)$ Let $x, y \in R$ such that $0 \neq x y \in P$ and suppose that $s x \notin P$. Thus, $x \notin(P: s)$. Since $y \in(P: x)$ and $x y \neq 0$, we get immediately, $(P: x) \subseteq(P: s)$. Hence, $y \in(P: s)$, and so $s y \in P$. Accordingly, $P$ is weakly $S$-prime.

$(2) \Rightarrow(3)$ Let $I$ and $J$ be two ideals of $R$ such that $I J \subseteq P$ and, for the element $s$ of (2), we have $s I \nsubseteq P$ and $s J \nsubseteq P$. We claim that $I J=0$. Let $x \in I \backslash(P: s)$. We have $x J \subseteq P$. Then, $J \subseteq(P: x)$. Since $J \nsubseteq(P: s)$, we get $J \subseteq(P: x)=(0: x)$. Hence, $x J=0$. Next, suppose that $x \in I \cap(P: s)$. Let $y \in J$. If $y \notin(P: s)$ then, as previously, we obtain $y I=0$, and so $y x=0$. If $y \in(P: s)$, consider $j \in J$ such that $s j \notin P$. Hence, $j \notin(P: s)$ and $y+j \notin(P: s)$. Hence, $x j=0$ and $x(y+j)=0$. Then $x y=0$. So, $x J=0$. Consequently, $I J=0$.

$(3) \Rightarrow(1)$ Let $a, b \in R$ with $0 \neq a b \in P$. Then, $0 \neq(a)(b) \subseteq P$. Hence, by hypothesis we have $s(a) \subseteq P$ or $s(b) \subseteq P$. Thus, $s a \in P$ or $s b \in P$, and so we conclude that $P$ is weakly $S$-prime of $R$.

Corollary 8 ([1], Theorem 3). For proper ideal $P$ of $R$, the following are equivalent:

1. $P$ is weakly prime. 
2. For each $x \notin P$ we have either $(P: x)=P$ or $(P: x)=(0: x)$.

3. For ideals $I$ and $J$ of $R$ with $0 \neq I J \subseteq P$, either $I \subseteq P$ or $J \subseteq P$.

Proof. Take $S=\{1\}$ in Theoerm7.

Proposition 9. Let $R$ be a ring, $S$ be a multiplicative subset of $R$, and $P$ an ideal of $R$. If $P$ is a weakly $S$-prime ideal that is not $S$-prime, then $s P \sqrt{0}=0$ for some $s \in S$.

Proof. By Theorem 7, there exists $s \in S$ such that for each $x \notin(P: s)$ we have either $(P: x) \subseteq(P: s)$ or $(P: x)=(0: x)$.

Let $a \in \sqrt{0}$. If $a \in(P: s)$ then $s a \in P$. Thus, by Proposition $4, s a P=0$. So, suppose that $a \notin(P: s)$. Then, $(P: a) \subseteq(P: s)$ or $(P: a)=(0: a)$. Since $P \subseteq(P: a)$, the case $(P: a)=(0: a)$ implies that $a P=0$, and then $s a P=0$. Hence, suppose that $(P: a) \subseteq(P: s)$. Let $n \geq 1$ be a minimal integer such that $a^{n}=0$. Then, $a^{n-1} \in(P: a) \subseteq(P: s)$. Therefore, $s a^{n-1} \in P$. Clearly, $n-1 \geq 1$ since $P \cap S=\emptyset$. If $s a^{n-1} \neq 0$ then $s a \in P$, a contradiction. Hence, $s a^{n-1}=0$. Let $j$ be minimal such that $s a^{j}=0$. Since $s a \neq 0$, we get that $j>1$.

Suppose that there exists $p \in P$ such that $s a p \neq 0$. We have $0 \neq s a\left(a^{j-1}+p\right)=$ $s a p \in P$. Then, $s\left(a^{j-1}+p\right) \in P$. Hence, $0 \neq s a^{j-1} \in P$. Hence, $s a \in P$, a contradiction. Thus, $s a P=0$. Consequently, $s P \sqrt{0}=0$.

Corollary 10 ([1], Theorem 4). Let $P$ be a weakly prime ideal of $R$. If $P$ is not prime, then $P \subseteq \sqrt{0}$ and $P \sqrt{0}=0$.

Proof. Take $S=\{1\}$.

Corollary 11. Let $R$ be a ring and $S$ a multiplicative subset of $R$. If $P$ and $Q$ are weakly $S$-prime ideals that are not $S$-prime, then $s P Q=0$ for some $s \in S$.

Proof. By Proposition4, we have $P, Q \subseteq \sqrt{0}$. Thus, by Proposition9 there is $s \in S$ such $s P Q \subseteq s P \sqrt{0}=0$.

Proposition 12. Let $R$ be a commutative ring and $S$ a multiplicative subset of $R$. If $P$ is a weakly $S$-prime ideal, then $S^{-1} P$ is a weakly prime ideal of $S^{-1} R$.

Proof. As $S \cap P=\emptyset$, we have that $S^{-1} P \neq S^{-1} R$. Let $0 \neq \frac{a}{s_{1}} \frac{b}{s_{2}} \in S^{-1} P$ where $a, b \in R$ and $s_{1}, s_{2} \in S$. Then, $\frac{a}{s_{1}} \frac{b}{s_{2}}=\frac{p}{s_{3}}$ for some $p \in P$ and $s_{3} \in S$. So, there is $t \in S$ such $0 \neq t s_{3} a b=t s_{1} s_{2} p \in P$. Since $P$ is weakly $S$-prime, there exists $s \in S$ such that $s s_{3} \in P$ or $0 \neq s a b \in P$. Thus $0 \neq s a b \in P$, 
since $s t s_{3} \notin P$. Hence, $0 \neq s^{2} a \in P$ or $s b \in P$, and so $s a \in P$ or $s b \in P$. This implies that $\frac{a}{s_{1}}=\frac{s a}{s s_{1}} \in S^{-1} P$ or $\frac{b}{s_{2}}=\frac{s b}{s s_{2}} \in S^{-1} P$, and so we have the desired result.

Let $R$ be a ring, $S \subseteq R$ be a multiplicative subset of $R$, and $P$ an ideal of $R$ with $P \cap S=\emptyset$. It is clear that if $(P: s)$ is a weakly prime ideal of $R$ for some $s \in S$, then $P$ is a weakly $S$-prime ideal. However, the converse implication is not true in general.

Example 13. Set $R:=\mathbb{Z} / 12 \mathbb{Z}$ and $S=\{\overline{3}, \overline{9}\}$. The ideal $(\overline{0})$ of $R$ is weakly $S$-prime, but $(\overline{0}: \overline{3})=(\overline{0}: \overline{9})=\{\overline{0}, \overline{4}\}$ is not weakly prime.

Proposition 14. Let $R$ be a ring, $S$ be a multiplicative subset of $R$ consisting of regular elements, and $P$ be an ideal of $R$ disjoint with $S$. Then the following assertions are equivalent:

1. $P$ is weakly $S$-prime of $R$.

2. $(P: s)$ is a weakly prime ideal of $R$ for some $s \in S$.

3. $S^{-1} P$ is a weakly prime ideal of $S^{-1} R$ and there is $s \in S$ such that $(P: t) \subseteq(P: s)$ for all $t \in S$.

4. $S^{-1} P$ is a weakly prime ideal of $S^{-1} R$ and $S^{-1} P \cap R=(P: s)$ for some $s \in S$.

Proof. Since $P$ is weakly $S$-prime, then there exist $s \in S$ such that for all $a, b \in R$ with $0 \neq a b \in P$, we have $s a \in P$ or $s b \in P$.

$(1) \Rightarrow(2)$ Let $0 \neq a b \in(P: s)$. Then, $0 \neq s a b \in P$. Hence, $0 \neq s^{2} a \in P$ or $s b \in P$. Thus $s a \in P$ or $s b \in P$, since $S \cap P=\emptyset$. Hence, we conclude that $a \in(P: s)$ or $b \in(P: s)$, and so $(P: s)$ is a weakly prime ideal of $R$.

$(1) \Rightarrow(3)$ By Proposition12, $S^{-1} P$ is a weakly prime ideal of $S^{-1} R$. Let $s \in S$ be the element associated to $P$. Let $t \in S$ and $0 \neq a \in(P: t)$. So, $0 \neq t a \in P$. Hence, st $\in P$ or $s a \in P$. Since $P \cap S=\emptyset$, st $\notin P$ which implies that $s a \in P$. Consequently, $a \in(P: s)$.

(1) $\Rightarrow(4)$ Again, $S^{-1} P$ is a weakly prime ideal of $S^{-1} R$ (by Proposition12). Let $0 \neq a \in(P: s)$. Then, $s a \in P$ and $a=\frac{a s}{s} \in S^{-1} P$. Hence, $a \in S^{-1} P \cap R$, and so $(P: s) \subseteq S^{-1} P \cap R$. Now, let $0 \neq a \in S^{-1} P \cap R$. Then, $a \in R$ and $a=\frac{p}{t}$ with $p \in P$ and $t \in S$. So, $0 \neq t a=p \in P$. Hence, st $\in P$ or $s a \in P$. Thus, $s a \in P$ since $S \cap P=\emptyset$. Consequently $a \in(P: s)$, and then $S^{-1} P \cap R \subseteq(P: s)$.

$(2) \Rightarrow(1)$ Clear. 
(3) $\Rightarrow(1)$ Let $a, b \in R$ such that $0 \neq a b \in P$. Since $0 \neq \frac{a}{1} \frac{b}{1} \in S^{-1} P$, we have $\frac{a}{1} \in S^{-1} P$ or $\frac{b}{1} \in S^{-1} P$. If $\frac{a}{1} \in S^{-1} P$, then $\frac{a}{1}=\frac{p}{t}$ for some $p \in P$ and $t \in S$. Hence, $t a=p$, and so $a \in(P: t) \subseteq(P: s)$. Similarly, if $\frac{b}{1} \in S^{-1} P$ then $s b \in P$, which means that $P$ is a weakly $S$-prime ideal of $R$.

(4) $\Rightarrow(1)$ Let $a, b \in R$ such that $0 \neq a b \in P$. Since $0 \neq \frac{a}{1} \frac{b}{1} \in S^{-1} P$, we have $\frac{a}{1} \in S^{-1} P$ or $\frac{b}{1} \in S^{-1} P$. If $\frac{a}{1} \in S^{-1} P$, then $\frac{a}{1}=\frac{p}{t}$ for some $p \in P$ and $t \in S$. Hence, $t a=p \in P$ and so $a=\frac{t a}{t} \in S^{-1} P \cap R$. Then, by hypothesis $a \in(P: s)$, that is $s a \in P$. Similarly, if $\frac{b}{1} \in S^{-1} P$ then $s b \in P$. Thus, $P$ is a weakly $S$-prime ideal of $R$.

Proposition 15. Let $R$ be a commutative ring and $S$ a multiplicative subset of $R$. Then the following assertions are equivalent:

1. (0) is the only weakly $S$-prime ideal of $R$.

2. (0) is the only $S$-prime ideal of $R$.

3. $R$ is a domain and $S^{-1} R$ is a field.

Proof. (1) $\Rightarrow(2)$ Clear.

$(2) \Rightarrow(3)$ By $[9$, Proposition 2.12], there is a prime ideal $P$ with $P \cap S=\emptyset$. Hence, $P$ is an $S$-prime ideal of $R$. Then, $P=(0)$, and so $R$ is a domain. Let $a \in R \backslash\{0\}$ and $s \in S$. We claim that $\frac{a}{s}$ is invertible in $S^{-1} R$. If $a \in S$ then we have the desired result. Hence, we may assume $a \notin S$. Suppose that $<a>\cap S=\emptyset$. Then, by [9, Proposition 2.12], there is a prime ideal $P$ of $R$ such that $\langle a\rangle \subseteq P$. Hence, $\langle a\rangle \subseteq P=\{0\}$, a contradiction. Thus, $<a>\cap S \neq \emptyset$. Let $s^{\prime} \in<a>\cap S$. Set $s^{\prime}=a t$. We have, $\frac{s t}{s^{\prime}} \in S^{-1} R$ and $\frac{a}{s} \cdot \frac{s t}{s^{\prime}}=\frac{a s t}{s s^{\prime}}=\frac{s s^{\prime}}{s s^{\prime}}=\frac{1}{1}$. Then, $\frac{a}{s}$ is invertible in $S^{-1} R$ as desired. So, $S^{-1} R$ is a field.

$(3) \Rightarrow(1)$ Let $P$ be a nonzero weakly $S$-prime ideal of $R$. Let $p \in P \backslash\{0\}$. Since $S^{-1} R$ is a field, there exists $a \in R \backslash\{0\}$ and $s \in S$ such that $\frac{p}{1} \cdot \frac{a}{s}=\frac{1}{1}$. Since $R$ is a domain, we deduce that $p a=s \in P \cap S=\emptyset$, a contradiction. Consequently, (0) is the only weakly $S$-prime ideal of $R$.

Proposition 16. Let $R$ be a ring, $S \subseteq R$ a multiplicative set and $P$ an ideal of $R$ disjoint with $S$. If $Q$ is an ideal of $R$ such that $Q \cap S \neq \emptyset$ and $P$ is a weakly $S$-prime, then $P \cap Q$ and $P Q$ are weakly $S$-prime ideals of $R$.

Proof. It is clear that $(P \cap Q) \cap S=\emptyset$ and $P Q \cap S=\emptyset$. First, we will prove that $P \cap Q$ is a weakly $S$-prime of $R$. Pick $s^{\prime} \in Q \cap S$ and let $a, b \in R$ such that $0 \neq a b \in P \cap Q \subseteq P$. Then, there is $s \in S$ such that $s a \in P$ or $s b \in P$. Thus, $s s^{\prime} a \in P \cap Q$ or $s s^{\prime} b \in P \cap Q$, and $s s^{\prime} \in S$. Consequently, $P \cap Q$ is a weakly $S$-prime ideal of $R$. The proof is similar for $P Q$. 
Proposition 17. Let $R$ be a commutative ring and $S$ a multiplicative subset of $R$. Then the following assertions are equivalent:

1. Every weakly $S$-prime ideal of $R$ is prime.

2. $R$ is a domain and every $S$-prime ideal of $R$ is prime.

Proof. (1) $\Rightarrow(2)$ Since (0) is weakly $S$-prime ideal of $R$, and every weakly $S$-prime ideal of $R$ is prime, $R$ is a domain.

$(2) \Rightarrow(1)$ As $R$ is domain, then every weakly $S$-prime ideal of $R$ is $S$-prime. Consequently, every weakly $S$-prime ideal of $R$ is prime.

Let $R$ be a commutative ring and $S$ a multiplicative subset of $R$. Recall from[1] that an ideal $I$ of $R$ is called $S$-finite (resp., $S$-principal) if $s I \subseteq J \subseteq I$ for some finitely generated (resp., principal) ideal $J$ of $R$ and some $s \in S$. Also, $R$ is called $S$-Noetherian (resp., $S$-PIR) if each ideal of $R$ is $S$-finite (resp., $S$-principal).

Theorem 18. Let $R$ be a ring and $S$ be a multiplicative subset of $R$. Then the following assertions are equivalent:

1. $R$ is $S$-Noetherian (resp., $S$-PIR).

2. Every weakly $S$-prime ideal of $R$ is $S$-finite (resp., $S$-principal).

3. Every $S$-prime ideal of $R$ is $S$-finite (resp., $S$-principal).

4. Every prime ideal of $R$ is $S$-finite (resp., $S$-principal).

Proof. (1) $\Rightarrow(2) \Rightarrow(3)$ Trivial.

$(3) \Rightarrow(4)$ Follows from [8, Theorem 3] ( the proof of "resp., $S$-principal" statement is analogue to that of $(2) \Rightarrow(3)$ in $[8$, Theorem 3]).

$(4) \Rightarrow(1)$ Follows from [1, Corollary 5] (resp., [1, Proposition 5]).

Remark 19. Let $S_{1} \subseteq S_{2}$ be multiplicative subsets of $R$ and $P$ an ideal of $R$ disjoint with $S_{2}$. Clearly, if $P$ is a weakly $S_{1}$-prime of $R$, then $P$ is weakly $S_{2}$-prime. However, the converse is not true general. To see that, consider the ideal $P=(4 X)$ of the rings $\mathbb{Z}[X]$ and set $S_{1}=\{1\}$ and $S_{2}=\left\{2^{n} \mid n \in \mathbb{N}\right\}$. By Example 3, $P$ is a weakly $S_{2}$-prime ideal of $\mathbb{Z}[X]$ but not weakly $S_{1}$-prime.

Proposition 20. Let $S_{1} \subseteq S_{2}$ be multiplicative subsets of $R$ such that for any $s \in S_{2}$, there is an element $t \in S_{2}$ satisfying st $\in S_{1}$. If $P$ is a weakly $S_{2}$-prime ideal of $R$, then $P$ is a weakly $S_{1}$-prime ideal of $R$. 
Proof. Let $a, b \in R$ such that $0 \neq a b \in P$. So, there is $s \in S_{2}$ such that $s a \in P$ or $s b \in P$. By the assumption, $s^{\prime}=s t \in S_{1}$ for some $t \in S_{2}$, and then $s^{\prime} a \in P$ or $s^{\prime} b \in P$. Consequently, $P$ is a weakly $S_{1}$-prime ideal of $R$.

Let $S$ be a multiplicative subset of $R, S^{*}=\left\{r \in R \mid \frac{r}{1}\right.$ is unit in $\left.S^{-1} R\right\}$ denotes the saturation of $S$. Note that, $S^{*}$ is a multiplicative subset containing $S$. A multiplicative subset $S$ of $R$ is called saturated if $S=S^{*}$. It is clear that $S^{*}$ is always a saturated multiplicative subset of $R$ [10].

Proposition 21. Let $R$ be a ring, $S$ be a multiplicative subset of $R$, and $P$ an ideal of $R$ disjoint with $S$. Then, $P$ is a weakly $S$-prime ideal of $R$ if and only if $P$ is a weakly $S^{*}$-prime ideal.

Proof. It is clear that $S^{*} \cap P=\emptyset$. We will show that for any $r \in S^{*}$, there is $r^{\prime} \in S^{*}$ such that $r r^{\prime} \in S$. Let $r \in S^{*}$, then $\frac{r}{1} \frac{a}{s}=1$ for some $s \in S$ and $a \in R$. This implies that $t a r=t s \in S$, for some $t \in S$. Now, take $r^{\prime}=t a$. Then, we have $r^{\prime} \in S^{*}$ with $r r^{\prime} \in S$, and so the desired condition is satisfied. Therefore, by putting $S=S_{1}$ and $S_{2}=S^{*}$, we conclude immediately the result from the proposition 20 .

Proposition 22. Let $f: R \rightarrow R^{\prime}$ be a ring homomorphism and $S$ a multiplicative subset of $R$. Then, the followings hold:

1. If $f$ is an epimorphism and $P$ is a weakly $S$-prime ideal of $R$ containing $\operatorname{Ker}(f)$, then $f(P)$ is a weakly $f(S)$-prime ideal of $R^{\prime}$.

2. If $f$ is a monomorphism and $P^{\prime}$ is a weakly $f(S)$-prime ideal of $R^{\prime}$, then $f^{-1}\left(P^{\prime}\right)$ is a weakly $S$-prime ideal of $R$.

Proof. (1) Let $r \in f(S) \cap f(P)$. Then, $r=f(p)=f(s)$ for some $p \in P$ and $s \in S$. So, $s-p \in \operatorname{Ker}(f) \subseteq P$, which implies that $s \in P$, a contradiction. Hence, $f(S) \cap f(P)=\emptyset$. Now, let $0 \neq a^{\prime} b^{\prime} \in f(P)$. Then, there is $a, b \in R$ such that $f(a)=a^{\prime}, f(b)=b^{\prime}$ and $0 \neq f(a b)=a^{\prime} b^{\prime} \in f(P)$. Since $\operatorname{Ker}(f) \subseteq P$, we get $0 \neq a b \in P$, and so $s a \in P$ or $s b \in P$ for some $s \in S$. It means that $f(s) a^{\prime} \in f(P)$ or $f(s) b^{\prime} \in f(P)$. Thus, $f(P)$ is weakly $f(S)$-prime ideal of $R^{\prime}$. (2) Let $P^{\prime}$ be an $f(S)$-prime ideal of $R^{\prime}$. So there exists $s \in S$ such that, for all $a^{\prime}, b^{\prime} \in R^{\prime}, 0 \neq a^{\prime} b^{\prime} \in P^{\prime}$ implies $f(s) a^{\prime} \in P^{\prime}$ or $f(s) b^{\prime} \in P^{\prime}$. The condition $f^{-1}\left(P^{\prime}\right) \cap S=\emptyset$ is trivial. Let $a, b \in R$ such that $0 \neq a b \in f^{-1}\left(P^{\prime}\right)$. Since $\operatorname{Ker}(f)=\{0\}$, we get $0 \neq f(a b)=f(a) f(b) \in P^{\prime}$. Then, $f(s) f(a)=f(s a) \in$ $P^{\prime}$ or $f(s) f(b)=f(s b) \in P^{\prime}$. Thus, $s a \in f^{-1}\left(P^{\prime}\right)$ or $s b \in f^{-1}\left(P^{\prime}\right)$, and so we conclude that $f^{-1}\left(P^{\prime}\right)$ is a weakly $S$-prime of $R$. 
Let $R$ be a ring, $S$ be a multiplicative subset of $R$, and $I$ an ideal of $R$ disjoint with $S$. Let $s \in S$, we denote by $\bar{s}$ the class of $s$ in $R / I$. Let $\bar{S}=\{\bar{s} \mid s \in S\}$. It is easy to check that $\bar{S}$ is a multiplicative subset of $R / I$.

Corollary 23. Let $R$ be a ring and $S$ be a multiplicative subset of $R$.

1. If $I \subseteq J$ be two ideals of $R$. If $J$ is a weakly $S$-prime of $R$, then $J / I$ is a weakly $\bar{S}$-prime of $R / I$.

2. If $R$ is sub-ring of $R^{\prime}$ and $J^{\prime}$ is a weakly $S$-prime of $R^{\prime}$, then $J^{\prime} \cap R$ is a weakly $S$-prime of $R$.

Proof. (1) Follows by applying Proposition22(1) to the canonical surjection $\pi: R \rightarrow R / I$.

(2) It suffices to apply Proposition22(2) to the naturel injection $\iota: R \hookrightarrow R^{\prime}$, since $\iota^{-1}\left(J^{\prime}\right)=J^{\prime} \cap R$.

Proposition 24. Let $R:=R_{1} \times R_{2}, S=S_{1} \times S_{2}$, where $S_{i}$ is a multiplicative subset of $R_{i}$ and $P_{1}$ and $P_{2}$ be an nonzero ideals of $R_{1}$ and $R_{2}$, respectively. Then the following are equivalent:

1. $P:=P_{1} \times P_{2}$ is a weakly $S$-prime of $R$.

2. $P_{1}$ is an $S_{1}$-prime ideal of $R_{1}$ and $S_{2} \cap P_{2} \neq \emptyset$ or $P_{2}$ is an $S_{2}$-prime ideal of $R_{2}$ and $S_{1} \cap P_{1} \neq \emptyset$.

3. $P:=P_{1} \times P_{2}$ is an $S$-prime of $R$.

Proof. (1) $\Rightarrow(2)$ Let $0_{R} \neq(p, q) \in P$ with $p \in R_{1}$ and $q \in R_{2}$. Then, $0 \neq(p, q)=(p, 1)(1, q) \in P$. Since, $P$ is weakly $S$-prime ideal of $R$, then there is $s=\left(s_{1}, s_{2}\right) \in S$ such that $s(p, 1)=\left(s_{1} p, s_{2}\right) \in P$ or $s(1, q)=\left(s_{1} 1, s_{2} q\right) \in P$. Thus, $S_{1} \cap P_{1} \neq \emptyset$ or $S_{2} \cap P_{2} \neq \emptyset$. Assume, $S_{2} \cap P_{2} \neq \emptyset$. As, $P \cap S=\emptyset$, we have $S_{1} \cap P_{1}=\emptyset$. Now, we show that $P_{1}$ is an $S_{1}$-prime ideal of $R_{1}$. Let $p p^{\prime} \in P_{1}$ for some $p, p^{\prime} \in R_{1}$. Since $S_{2} \cap P_{2} \neq \emptyset$, then there is $0_{R_{2}} \neq t \in S_{2} \cap P_{2}$, and so we have $0_{R} \neq(p, t)\left(p^{\prime}, 1\right) \in P$. Hence, $s(p, t)=\left(s_{1} p, s_{2} t\right) \in P$ or $s\left(p^{\prime}, 1\right)=\left(s_{1} p^{\prime}, s_{2}\right) \in P$. So, we get $s_{1} p \in P_{1}$ or $s_{1} p^{\prime} \in P_{1}$, as desired.

$(2) \Rightarrow(3)$ Follows directly from [7, Lemma 2.13].

$(3) \Rightarrow(1)$ Clear.

Proposition 25. Let $R:=R_{1} \times R_{2}$ and $S=S_{1} \times S_{2}$, where $S_{i}$ is a multiplicative subset of $R_{i}$. Then the following are equivalent.

1. Every proper ideal of $R$ is weakly $S$-prime.

2. $R_{1}$ and $R_{2}$ are fields. 
Proof. (1) $\Rightarrow(2)$ Let $I_{1}$ be a proper ideal of $R_{1}$. Then, $I_{1} \times R_{2}$ is a weakly $S$-prime ideal of $R$, and so $I_{1}$ is an $S_{1}$-prime ideal of $R_{1}$ (by Proposition 24). Thus, every proper ideal of $R_{1}$ is $S_{1}$-prime. So, by [7, Corollary 2.27], $R_{1}$ is a field. Similarly, $R_{2}$ is a field.

$(2) \Rightarrow(1)$ Since $R_{1}$ and $R_{2}$ are fields. Then, we have exactly three proper ideals

$\{0\} \times\{0\},\{0\} \times R_{2}$, and $R_{1} \times\{0\}$ which are $S$-prime (by Proposition24).

Proposition 26. Let $R$ be a commutative ring and $S \subseteq R$ a multiplicative set. Then the following are equivalent.

1. Every proper ideal of $R$ is weakly $S$-prime.

2. $S \subseteq U(R)$ and " $(R, \mathfrak{m})$ is a quasilocal ring (possibly a field) with $\mathfrak{m}^{2}=0$ " or " $R=F_{1} \times F_{2}$ where $F_{1}$ and $F_{2}$ are fields".

Proof. Suppose that (1) holds and let $s \in S$. Since every maximal ideal of $R$ is weakly $S$-prime by hypothesis, then there is no maximal ideal of $R$ which contains $s$. This implies that $s$ is a unit element of $R$, and so $S \subseteq \mathrm{U}(R)$.

Hence, if (1) or (2) holds, $S \subseteq \mathrm{U}(R)$. In this situation, the concepts of weakly $S$-prime ideals and weakly prime ideals coincide, and the result follows directly from [1, Theorem 8].

Let $R$ be a ring and $M$ be an $R$-module. The additive group $R \times M$ with multiplication $(a, m)\left(b, m^{\prime}\right)=\left(a b, a m^{\prime}+b m\right)$ is a commutative ring with identity, noted $R \propto M$ and called the Nagata idealization. If $P$ is an ideal of $R$ then, $P \propto M$ is an ideal of $R \propto M$. Also, if $S$ is a multiplicative subset of $R$ then $S \propto 0$ and $S \propto M$ are multiplicative subset of $R \propto M$.

Proposition 27. Let $R$ be a ring, $M$ be an $R$-module, $S$ be a multiplicative subset of $R$ and $P$ be an ideal of $R$ disjoint with $S$. Then the following are equivalent:

1. $P \propto M$ is a weakly $(S \propto M)$-prime ideal of $R \propto M$.

2. $P \propto M$ is a weakly $(S \propto 0)$-prime ideal of $R \propto M$.

3. $P$ is a weakly $S$-prime of $R$ associated to $s \in S$, and if $a, b \in R$ with $a b=0$, but $s a \notin P$ and $s b \notin P$ then $a \in \operatorname{ann}_{R}(M)$ and $b \in \operatorname{ann}_{R}(M)$.

Proof. (2) $\Rightarrow$ (1) Follows from Remark19 since $S \propto 0 \subseteq S \propto M$.

$(1) \Rightarrow(3)$ Let $a, b \in R$ such that $0 \neq a b \in P$. Then, $(0,0) \neq(a, 0)(b, 0) \in$ $P \propto M$. As $P \propto M$ is a weakly $(S \propto M)$-prime ideal of $R \propto M$, there is $(s, n) \in S \propto M$ such that $(s, n)(a, 0)=(s a, a n) \in P \propto M$ or $(s, n)(b, 0)=$ $(s b, b n) \in P \propto M$. Thus, $s a \in P$ or $s b \in P$, and so $P$ is a weakly $S$-prime 
of $R$. Now, suppose that $a b=0$, but $s a \notin P$ and $s b \notin P$. Assume that $a \notin \operatorname{ann}_{R}(M)$. Then, there is $m \in M$ such that $a m \neq 0$, and so we have $(0,0) \neq(a, 0)(b, m) \in P \propto M$. Hence, $(s, n)(a, 0)=(s a, a n) \in P \propto M$ or $(s, n)(b, m)=(s b, s m+b n) \in P \propto M$, a contradiction.

(3) $\Rightarrow(2)$ Let $(0,0) \neq(a, m)(b, n) \in P \propto M$, where $(a, m),(b, n) \in R \propto M$. If $a b \neq 0$, then $s a \in P$ or $s b \in P$, and hence $(s, 0)(a, m) \in P \propto M$ or $(s, 0)(b, n) \in P \propto M$. Assume that $a b=0$, but $s a \notin P$ and $s b \notin P$. Then $a$, $b \in \operatorname{ann}_{R}(M)$. Consequently, we get $(a, m)(b, n)=(0,0)$, a contradiction.

Let $R$ be a ring and $I$ be an ideal of $R$. The amalgamated duplication of $R$ along $I$, denoted by $R \bowtie I$, is the subring of $R \times R$ given by $A \bowtie I=\{(a, a+i \mid a \in I, i \in I\}$. This construction was introduced and its basic properties were studied by D'Anna and Fontana in $[4,5]$ and then it was investigated by D'Anna in [3] with the aim of applying it to curve singularities (over algebraic closed fields) where he proved that the amalgamated duplication of an algebroid curve along a regular canonical ideal yields a Gorenstein algebroid curve.

Recently, the authors in [6] studied some kind of weakly prime ideals in amalgamated algebras (a generalization of a the duplication construction). In the next result of this paper, we initiate the study of weakly $S$-prime ideals over amalgamated duplication of a ring along an ideal.

Proposition 28. Let $R$ be a ring, $I$ be an $R$-module, $S$ be a multiplicative subset of $R$, and $P$ be an ideal of $R$ disjoint with $S$. Then the following are equivalent:

1. $P \bowtie I$ is a weakly $(S \bowtie I)$-prime ideal of $R \bowtie I$.

2. $P \bowtie I$ is a weakly $(S \bowtie 0)$-prime ideal of $R \bowtie I$.

3. $\quad P$ is a weakly $S$-prime of $R$ associated to $s \in S$, and

- if there exists $a, b \in R$ with $a b=0$, but $s a \notin P$ and $s b \notin P$ then $a \in \operatorname{ann}_{R}(I), b \in \operatorname{ann}_{R}(I)$, and $I^{2}=(0)$.

Proof. (2) $\Rightarrow$ (1) Follows from Remark19 since $S \bowtie 0 \subseteq S \bowtie I$.

$(1) \Rightarrow(3)$ Suppose that $P \bowtie I$ is a weakly $(S \bowtie I)$-prime ideal of $R \bowtie I$ associated to $(s, s+i)$. Let $a, b \in R$ such that $0 \neq a b \in P$. Then, $(0,0) \neq$ $(a, a)(b, b) \in P \bowtie I$. Thus, $(s, s+i)(a, a) \in P \bowtie I$ or $(s, s+i)(b, b) \in P \bowtie I$. So, $s a \in P$ or $s b \in P$, and so $P$ is a weakly $S$-prime of $R$ associated to $s$. Now, suppose that there exists $a, b \in R$ such that $a b=0$ but $s a \notin P$ and $s b \notin P$. Assume that $a \notin \operatorname{ann}_{R}(I)$. Then, there is $j \in I$ such that $a j \neq 0$, and so we have $(0,0) \neq(a, a)(b, b+j) \in P \bowtie I$. Hence, $(s, s+i)(a, a)=(s a, s a+i a) \in$ $P \bowtie I$ or $(s, s+i)(b, b+j)=(s b, s b+i b+s j+i j) \in P \bowtie I$, a contradiction. 
Hence, $a \in \operatorname{ann}_{R}(I)$. Similarly, $b \in \operatorname{ann}_{R}(I)$. Let $j, k \in I$. We have $(a, a+$ $j)(b, b+k)=(0, j k) \in P \bowtie I$. If $j k \neq 0$ then $(a, a+j)(b, b+k) \neq(0,0)$. Thus, $(s, s+i)(a, a)=(s a, s a) \in P \bowtie I$ or $(s, s+i)(b, b+j)=(s b, s b+i j) \in P \bowtie I$, a contradiction. Thus, $I^{2}=(0)$.

$(3) \Rightarrow(2)$ Let $(0,0) \neq(a, a+j)(b, b+k) \in P \bowtie I$, where $(a, a+j),(b, b+k) \in$ $R \bowtie I$. If $a b \neq 0$, then $s a \in P$ or $s b \in P$, and hence $(s, s)(a, a+j) \in P \bowtie I$ or $(s, s)(b, b+k) \in P \bowtie I$. Assume that $a b=0$, but $s a \notin P$ and $s b \notin P$. Then $a, b \in \operatorname{ann}_{R}(I)$. Consequently, we get $(a, a+j)(b, b+k)=(0,0)$, a contradiction.

\section{Acknowledgments}

The authors extend their appreciation to the Deanship of Scientific Research at King Khalid University for funding this work through research groups program under Grant number R.G.P.1/178/41. The authors would like also to thank the referee for careful reading of the manuscript.

\section{References}

[1] D.D. Anderson, E. Smith, Weakly prime ideals, Houston J. Math. 29 (2003) 831840.

[2] A. Badawi, U. Tekir and E. Yetkin, On 2-absorbing primary ideals in commutative rings, Bull. Korean Math. Soc., 51(4) (2014), 1163-1173.

[3] M. D'Anna, A construction of Gorenstein rings, J. Algebra 306(6) (2006), $507-519$.

[4] M. D'Anna and M. Fontana, An amalgamated duplication of a ring along an ideal: the basic properties, J. Algebra Appl. 6(3) (2007), 443-459.

[5] M. D'Anna and M. Fontana, The amalgamated duplication of a ring along a multiplicative-canonical ideal, Ark. Mat. 45(2) (2007), 241-252.

[6] N. Mahdou, M. A. S. Moutui, Y. Zahir, Weakly prime ideals issued from an amalgamatedalgebra, Hacet. J. Math. Stat. 49 (3) (2020), 1159-1167.

[7] E. S. Sevim, I. T Arabac, U. Tekir and S. Koc, On S-prime submodules. Turkish J. Math. (2019) 43: 1036-1046.

[8] A. Hamed and A. Malek, $S$-prime ideals of a commutative ring. Beitr. Algebra Geom. (2019). 
[9] M. D.Larsen and P. J. McCarthy, Multiplicative theory of ideals. New York: Academic Press. (1971).

[10] R. W. Gilmer, Multiplicative Ideal Theory, Vol. 12 (M. Dekker, 1972).

Fuad ali Ahmed ALMAHDI,

Department of Mathematics, Faculty of Science,

King Khalid University,

P.O. Box. 9004, Abha, Saudi Arabia.

Email: fuadalialmahdy@hotmail.com

El Mehdi BOUBA,

Team of Modeling and Scientific Computing,

Mathematics Department, Pluridisciplinary faculty,

Mohammed First University,

B.P. 300, Selouane, Nador 62700, Morocco.

Email: mehdi8bouba@hotmail.fr

Mohammed TAMEKKANTE,

Department of Mathematics, Faculty of Science,

University Moulay Ismail Meknes,

Box 11201, Zitoune, Morocco.

Email: tamekkante@yahoo.fr 